\title{
Una nueva especie ecuatoriana del género de peces andinos Grundulus (Characiformes: Characidae)
}

\author{
César Román-Valencia ${ }^{1}$, Raquel I. Ruiz C. ${ }^{1} \&$ Ramiro Barriga ${ }^{2}$ \\ 1 Universidad del Quindio, laboratorio de ictiología, A.A. 460, Armenia, Quindio, Colombia. \\ croman@uniquindio.edu.co \& zutana_1@yahoo.com \\ 2 Escuela Politécnica Nacional, Depto. de Ciencias Biológicas, Museo de Zoología, Casilla 17-01-2759, Quito, Ecuador. \\ rbarriga@server.epn.edu.ec \\ Recibido 04-VII-2005. Corregido 11-VII-2005. Aceptado 10-VIII-2005.
}

\begin{abstract}
A new Ecuadorian species of the Andean fish genus Grundulus (Characiformes: Characidae). The new species Grundulus quitoensis is described from El Voladero Lake, El Angel Biological Reserve, Northern Ecuador. The new taxon can be distinguished from all congeners by the following characters, respectively: a low number of unbranched rays in the anal fin (three vs. four-five) and in pectoral fins (on vs. two - three), a higher number of dentary teeth (12-14 vs. 8-10), a second infraorbital about three times larger than the first (vs. about two times larger), a third infraorbital which is not in contact with the preopercle (vs. in contact with preopercle), the presence of four small fenestrae in the infero-lateral bone dentary (vs. one - two); and a premaxilla which presents a long lateral process (vs. short), a maxilla with two notches in the infero-lateral surface (vs. one notch in the infero-lateral surface). Rev. Biol. Trop. 53(3-4): 537-544. Epub 2005 Oct 3.
\end{abstract}

Key words: Grundulus quitoensis sp. nov. Characidae, Andean fish, South America.

Grundulus es un género de Characidae de pequeño porte conocido hasta el momento para los Andes de Colombia. Presenta, entre otros, los siguientes caracteres diagnósticos: ausencia de aleta adiposa; número reducido de escamas perforadas en la línea lateral (0-8); radios en la aleta anal iv-v, 11-12; premaxilar, y dentario sólo con dientes cónicos (RománValencia et al. 2003). Se ha descrito dos especies: G. bogotensis (Humboldt y Valenciennes 1833) endémica de la Sabana de Bogotá (Cala y Román-Valencia 1994, Román-Valencia et al. 2003) y G. cochae de la Laguna de la Cocha al sur de Colombia (Román-Valencia et al. 2003). Tradicionalmente, el género Grundulus es incluido dentro de la subfamilia Cheirodontinae (Eigenmann 1915, 1922, Dahl 1971, Miles 1971, Géry 1977), aunque recientes estudios indican que el género carece de los caracteres diagnósticos de la subfamilia Cheirodontinae (Malabarba 1998, Weitzman y Malabarba 1999,
Román-Valencia et al. 2003). Su relación filogenética dentro de los Characidae es desconocida y el género es de posición incierta dentro de la familia (Malabarba 1998, Weitzman y Malabarba 1999).

La captura y el examen de ejemplares de Grundulus en la Laguna El Voladero, reserva biológica El Angel al norte de Ecuador, mostró que pertenecen a una especie no descrita perteneciente al género Grundulus. Por consiguiente, el propósito del presente artículo es describir la nueva especie.

\section{MATERIALES Y MÉTODOS}

El material fue fijado en formol al $10 \%$ y luego preservado en alcohol etílico al 73\%, se depositó en el laboratorio de ictiología, Universidad del Quindío, Armenia, Colombia (IUQ); museo de Zoología, Departamento 
de Ciencias Biológicas, Escuela Politécnica Nacional, Quito, Ecuador (MEPN); en el Museo de Ciencias Naturales de la Universidad Experimental de los Llanos Occidentales, UNELLEZ- Guanare, Venezuela (MCNG), y en United States National Museum, Smithsonian Institution, Washington (USNM).

Las medidas de los ejemplares se tomaron con un calibrador digital, hasta centésimas de milímetro. Todas las medidas fueron obtenidas punto a punto, i.e. no ortogonal al eje principal del cuerpo. Los recuentos de radios, escamas y dientes empleando estereoscopio y aguja de disección. Las medidas y recuentos (Cuadro 1) se realizaron sobre el lado izquierdo de los ejemplares, excepto cuando éstos estaban deteriorados en tal lado. Medidas y conteos sigue a Hubbs y Lagler (1958) y a Weitzman y Malabarba (1999).

Los 21 caracteres morfométricos utilizados en éste trabajo (Cuadro 1) fueron útiles para efectuar un análisis de componentes principales (ACP). En éste caso, se utilizó el paquete Past, versión 1.29 bajo Windows (disponible en: htt://folk.uio.no/ohammer/past/).

Las observaciones de las estructuras óseas y cartílagos se hicieron sobre ejemplares clareados y teñidos de acuerdo a modificaciones al método descrito por Taylor y Van Dyke
(1985). La nomenclatura de huesos se basó en Weitzman (1962) y Vari (1995). El conteo y las determinaciones de vértebras se hizo con material diafanizado e incluye las cuatro primeras vértebras del aparato de weber.

\section{RESULTADOS}

Grundulus quitoensis sp.nov.

(Cuadro 1 y Fig. 1)

Holotipo: MEPN 1637, Ecuador, provincia de Carchi, lagunas El Voladero reserva

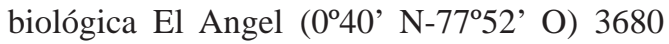
m.s.n.m., julio 20 de 2001, col. R. Barriga.

Paratipos: MEPN 001550, 10 ejemplares colectados con el holotipo; MCNG 53445, tres ejemplares; IUQ 532, dos ejemplares colectados con el holotipo.

No tipo: USNM 360016, ocho ejemplares, Lagunas El Voladero, julio 12 de 1998, col. E. Ternaus (cf. Barriga y Ternaus, 2005).

Diagnosis: El nuevo taxon se puede distinguir de las demás especies descritas en Grundulus por el menor número de radios

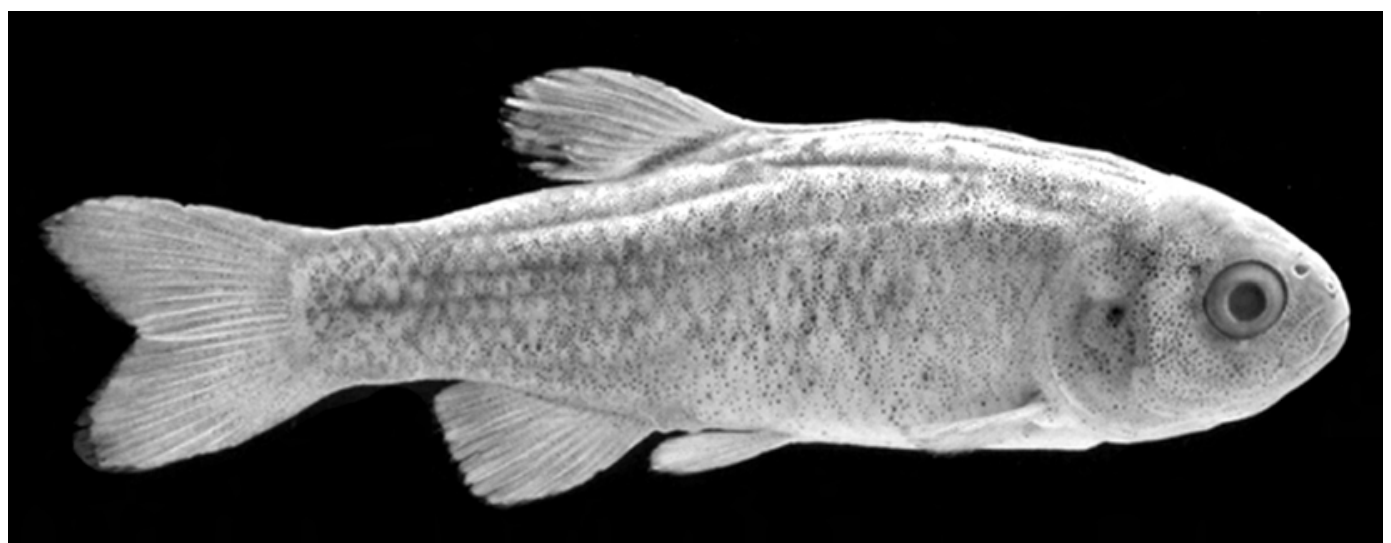

Fig. 1. Grundulus quitoensis sp nov. Paratipo: MCNG, Ecuador, provincia de Carchi, lagunas El Voladero, Reserva Biológica El Angel.

Fig. 1. Grundulus quitoensis sp.nov. Paratype: MCNG, Ecuador, Carchi Province, El Voladero lake, El Angel Biological Reserve. 
CUADRO 1

Datos morfométricos y merísticos de Grundulus quitoensis sp. nov.

TABLE 1

Morphometric and meristic data of Grundulus quitoensis sp. nov.

Morfometría:

Longitud estándar

Longitud total

Porcentaje de la longitud estándar:

1. Profundidad del cuerpo

2. Longitud hocico - aleta dorsal

3. Longitud hocico-aleta pectoral

4. Longitud hocico - aletas pélvicas

5. Longitud hocico-aleta anal

6. Longitud aleta dorsal- hipurales

7. Longitud aleta dorsal

8. Longitud aletas pectorales

9. Longitud aletas pélvicas

10.Longitud aleta anal

11. Longitud aleta dorsal - aleta anal

12. Longitud aleta dorsal - aleta pectoral

13. Profundidad del pedúnculo caudal

14. Longitud pedúnculo caudal

15. Longitud cabeza

Porcentaje de la longitud cabeza:

16. Longitud hocico

17. Diámetro del ojo

18. Longitud postorbital de la cabeza

19. Longitud del hueso maxilar

20. Ancho interorbital

21. Longitud mandíbula superior

Merística:

Número de escamas en la línea lateral

Número de radios en la aleta anal

Número de radios en las aletas pélvicas

Número de radios en las aletas pectorales

Número de radios en la aleta dorsal

$$
\text { Paratipos }
$$$$
\mathrm{n}=15
$$

23.41-51.09 (38.80)

47.83

28.64-62.47 (47.53)

58.02

21.23-32.46 (27.97)

31.09

57.07-62.74 (59.96) $\quad 61.43$

26.66-31.05 (28.72) $\quad 29.52$

$51.21-63.26(54.06) \quad 52.02$

63.3-70.98 (68.10) $\quad 69.31$

42.00-47.51 (44.34) $\quad 43.28$

18.59-25.05 (22.42) 22.73

$12.00-17.08(15.16) \quad 16.06$

$11.67-16.84(14.03) \quad 14.32$

$13.14-22.00(17.70) \quad 18.15$

23.75-34.71 (29.58) $\quad 32.45$

37.08-44.32 (40.89) $\quad 44.32$

$10.55-15.28(13.33) \quad 15.28$

$11.25-17.90(13.80) \quad 13.71$

$25.13-28.92(26.83) \quad 25.13$

19.65-28.29 (25.06) 26.96

$23.02-37.49(29.08) \quad 26.29$

34.27-49.11 (44.38) $\quad 45.26$

$21.45-32.11(26.6) \quad 28.70$

28.35-34.92 (31.29) $\quad 34.53$

$20.25-33.93(27.60) \quad 23.04$

$\begin{array}{cc}0-3 & 0 \\ \text { iii, } 11-13 & \text { iii,11 } \\ \text { ii,6 } & \text { ii,6 } \\ \text { i, 12-16 } & \text { i,13 } \\ \text { ii,8-9 } & \text { ii,9 }\end{array}$

Longitudes estándar y total en mm. Promedios entre paréntesis Standard and total length in mm. Average in parenthesis. 
simples en la aleta anal (tres vs. cuatro a cinco) y de radios simples en la aletas pectorales (uno vs. dos a tres), por el mayor número de dientes en cada dentario (12-14 vs. 8-10), perfil ventral posterior a la cintura pectoral hasta el origen de la aleta anal plano (vs. curvo), el segundo infraorbital es tres veces más grande que el primero (vs. dos veces más grande), el tercer infraorbital está separado del preopérculo (vs. en contacto con la superficie del preoperculo), el dentario con cuatro pequeñas fenestras en el margen infero-lateral (vs. uno a dos), el premaxilar presenta un proceso lateral largo (vs. corto), maxilar con dos muescas en la superficie infero-lateral (vs. maxilar con una muesca en la superficie inferolateral).

English diagnosis: The new taxon can be distinguished from all congeners by the low number of simple rays in the anal fins (three vs. four-five) and in pectoral fins (one vs. two-three), the higher number of dentary teeth (12-14 vs. 8-10), the second infraorbital which is three times larger than the first (vs. two times larger), the third infraorbital which is not in contact with the preopercle (vs. in contact with preopercle), the presence of four small fenestras in the infero-lateral bone (vs. one - two), a premaxilla which presents a long lateral process (vs. short), a maxilla with two notches in the infero-lateral surface (vs. one notch in the infero-lateral surface).

Descripción: Perfil dorsal de la cabeza en línea curva. Perfil ventral del hocico hasta la base de la cintura pectoral curvo, posterior a ésta hasta el origen de la aleta anal plano.

Osteología: Huesos infraorbitales osificados cubren el margen inferior de la órbita, por lo cual la parte superior del hiomandibular se encuentra descubierta, la espina del esfenótico se extiende lateralmente, prolonga el sistema latero sensorial del supraorbital al canal del pterótico. El primer infraorbital se extiende entre el lateroetmoide y el maxilar. El segundo infraorbital es tres veces más grande que el primero y cubre la región postero dorsal del articular. El tercer infraorbital está separado del preopérculo. Supraorbital presente.

Opérculo largo, plano o laminar y ubicado en la parte latero-posterior del neurocráneo, se articula al hiomandibular por una banda de cartílago. El subopérculo es una leve hoja de hueso parcialmente ampliada a lo largo del margen interna e inferior del opérculo. El interopérculo es delgado y se ubica anterior al subopérculo e inferior al preopérculo. El extremo anterior del preopérculo encaja en una ranura sobre la parte postero-ventral del cuadrado, mientras que la parte dorsal se sitúa entre el borde posterior del hiomandibular y el opérculo. La superficie lateral del preopérculo no contiene canales laterosensoriales.

Mandíbula inferior con una serie de 12 a 14 dientes de diferente tamaño a cada lado de los dentarios, en línea recta y sin espacio alguno entre éstos. Todos los dientes son cónicos. Dentario presenta cuatro pequeñas fenestras en el margen infero-lateral.

El premaxilar presenta un proceso lateral largo y presenta de dos a tres forámenes con una hilera de dientes cónicos sobre cada uno. Siete a ocho dientes en cada lado, orientados en línea recta. El maxilar es corto y el extremo posterior no alcanza hasta el extremo inferior de los dentarios. Maxilar con cinco a siete dientes cónicos de diferente longitud, ubicados en el borde supero-anterior. Maxilar con un proceso doblado en su extremo superior que se articula al proceso lateral del premaxilar y con dos muescas en la superficie infero-lateral.

El hueso mesetmoide es estrecho, se proyecta anterior de los frontales. Anteriormente el mesetmoide termina en dos procesos óseos que sostienen los procesos ascendentes del premaxilar y el extremo anterior se proyecta entre los procesos ascendentes del premaxilar. El prevomer es óseo, bordeado por una banda de cartílago que lo articula anteriormente al mesetmoide y lateralmente al palatino, el cual carece de dientes, es laminar, corto, y no se une al maxilar ni al premaxilar. El latero-etmoide es lateralmente angosto y ventralmente cóncavo, cubre el margen anterior de cada órbita. 
Los frontales están separados por la fontanela fronto-parietal y en su parte media se encuentra conectados por la barra epifiseal. Fontanela fronto-parietal angosta a nivel del extremo anterior, ancha en la parte posterior de los frontales en el ámbito de los parietales y en unión con el supraoccipital. Los dos parietales son cortos y se pliegan posteriormente sobre los procesos laterales del supraoccipital. Estos parietales se pliegan lateralmente al canal del pterótico y atravesados inferomedial por el canal parietal en una estructura cartilaginosa, que se extiende inferiormente hacia la fosa postemporal.

$\mathrm{El}$ arco del exoccipital se extiende internamente hacia la parte basal del supraoccipital, al cual se une por una banda de cartílago. El epioccipital es la estructura lateroposterior del neurocráneo, ventro-medial está rodeado por el exoccipital. Se extiende de forma arqueada, desde el extremo infero-posterior del pterótico hacia el supraoccipital, desde la parte media se extiende una apófisis hacia el extremo posterolateral del parietal.

El basioccipital está unido anteriormente al proótico, ventromedialmente al paraesfenoides y dorsalmente al exooccipital. Todas las uniones, excepto con el paraesfenoides, son sincrónicas. El proceso superior del extraescapular se extiende por encima del epioccipital. El pterótico es una estructura ósea latero supero posterior del neurocráneo, corta. Se une posteriormente al borde antero-medial del extraescapular, articulado anteriormente al esfenótico por una banda de cartílago. El esfenótico está debajo de los parietales, no cubierto por los infraorbitales, comunica el canal latero sensorial del supraorbital con el canal del pterótico, por medio del extremo inferior, que se extiende en una espina hacia el extremo posterior del supraorbital.

La región supero-medial de la órbita está ocupada por el pteroesfenoide con el orbitoesfenoide, unidos por una banda de cartílago. Lateralmente el pteroesfenoide y el orbitoesfenoide están plegados al margen lateral del frontal, atravesados medialmente por la cavidad craneal que se extiende posteriormente hacia el proótico y exooccipital, desemboca en la fosa postemporal. Se observan dos forámenes sobre la superficie infero medial del orbitosfenoide. El rinoesfenoides es una estructura medial del neurocráneo, se pliega sobre el borde anterior del pteroesfenoide por medio de una banda de cartílago. El rinoesfenoides es cartilaginoso, se extiende antero-lateralmente sobre la superficie anterior del paraesfenoides. El paraesfenoides es delgado, alargado, se ubica en la parte infero medial del branquiocráneo y no se encuentra dividido. La parte anterior cubre la superficie inferior del prevomer; la parte posterior se extiende sobre la superficie inferior del proótico, se extiende por debajo del margen inferior del basioccipital y antes de éste hueso se bifurca en dos procesos, que se extienden sobre el borde antero-medial del proótico, dando origen a un forámen. No se observan huesos nasales.

La parte superior del hiomandibular es de forma ondulada. Su apófisis posterior es corta y se pliega sobre el borde supero-anterior del preopérculo. El cuadrado cubre la superficie dorsal del preopérculo y posee tres brazos. Uno superior que se proyecta hacia el metapterigoide y es óseo. Los dos brazos posteriores son delgados se proyectan arriba y abajo del simpléctico. El cuadrado se une al metapterigoides a través de bandas de cartílago. La parte posterior del metapterigoides es ondulada.

El borde posterior del metapterigoide en contacto con la superficie dorso posterior del simpléctico y de la parte anterior del hiomandibular. El borde anterior del metapterigoide en contacto con el cuadrado y el mesopterigoide, por medio de una banda de cartílago que se extiende de forma ascendente en medio de la unión del mesopterigoide y el ectopterigoide. El mesopterigoide y ectopterigoide sin dientes, se proyectan desde el margen inferior del palatino. El extremo anterior del palatino se observa cartilaginoso, alcanza el ángulo formado por la apófisis del maxilar, y es paralelo al margen supero-dorsal del proceso dorsal del maxilar.

En el aparato de Weber, el centro de la primera vértebra es un disco simple sin depresiones dorsolaterales para la recepción del proceso articular ventral del escafium. La segunda vértebra tiene un proceso lateral alargado. La 
cuarta vértebra tiene un proceso lateral laminar: estrecho en su base y ancho en el resto de su superficie, el cual se extiende fuera de la parte media. El complejo neural plegado sobre el margen posterior del arco exooccipital y el supraoccipital, posteriormente se extiende sobre el margen superior de la cuarta vértebra, sobre el borde posterior del arco exooccipital y del supraoccipital. La superficie lateral presenta de cinco a seis forámenes. El claustrom es una estructura corta, angosta, delgada o reducida, se continúa inferiormente con el escafium, que se extiende de forma curva sobre la superficie dorso-medial del intercalarium y la superficie dorsal del disco basal de la primera y segunda vértebra.

Las vértebras precaudales se observan en número de 19 e incluyen las cuatro vértebras del aparato de weber. Cada una de estas vértebras tiene dos precigoapófisis neurales, un arco neural y una espina neural. Poseen postcigoapófisis hemales. Posee 16 vértebras caudales, dorsalmente cada vértebra registra una espina neural, un arco neural y una espina precigoapófisis neural y dos postcigoapófisis neurales. Infero-anterior de cada vértebra posee una espina hemal y un arco hemal. Los pre y postcigoapófisis se notan en todas las vértebras. Las últimas tres vértebras caudales sostienen la aleta caudal. Se observan de cuatro a cinco hipurales asociados con la última vértebra y el urostilo, el borde posterior de cada uno de los hipurales presenta una banda de cartílago. Hay 8/10 radios principales. El uroneural óseo se extiende de forma oblicua sobre los cinco primeros hipurales. En la parte dorsal del urostilo se localiza el proceso neural especializado, el cual es laminar, se articula el epural. En el dorso del urostilo hay un epural, se extiende sobre el borde posterior de los radios procurrentes. No hay espinas hipurales, proyecciones epineurales y ni epipleurales.

Aleta dorsal con margen anterior oblicuo y borde posterior redondeado. El componente proximal y medial de los primeros pterigióforos insertados entre las espinas neurales de las vértebras precaudales $15-18$. Todos los radiales proximales sin quilla. No se observa aleta adiposa.
La cintura pectoral se articula superiormente al extraescapular por el postemporal, estructura alargada y plegada sobre la superficie interna del cleitro, alargada y curva, se ubica posterior al opérculo. Sobre la superficie ventral del cleitro se pliega el supracleitro. El supracleitro se extiende anterior en un proceso alargado por debajo de la placa faríngea inferior del aparato branquial. La cara media es la superficie para la recepción de la parte lateral de la escápula. El cleitro es una estructura ovalada en su parte postero-lateral, plegada al margen inferior del supracleitrum. La escápula es una estructura ósea plegada al cleitro y coracoides por una banda de cartílago y con dos o tres forámenes, sobre el borde posterior se unen los radios proximales que sostienen los pterigióforos. La escápula se une al mesocoracoide por una banda de cartílago. El mesocoracoide es una estructura ósea que se extiende sobre la superficie exterior del supracleitrum. Presenta de seis a ocho forámenes. Hay tres radiales proximales. El coracoides es una estructura horizontalmente alargada, plegada como una quilla a la superficie interna del cleitro y supracleitro.

El margen lateral de las aletas pélvicas es oblicuo, alcanza el origen de la aleta anal. El hueso pélvico es una estructura corta. La región posterior está en contacto con los radios $\mathrm{y}$ es ancho, se proyecta interna hacia el proceso ísquico. El borde postero-lateral posee una banda de cartílago. Sobre el borde posterior del hueso pélvico, se extienden tres elementos radiales que sostiene los radios pélvicos, óseos, con una estructura distal cartilaginosa. La región anterior es puntiaguda, angosta y posee cartílago en su extremo. El proceso ísquico es alargado y curva que se extiende por detrás de los radios pélvicos, presenta en su extremo posterior una banda de cartílago. No presenta forámen.

En la aleta anal los dos primeros pterigióforos proximales asociados con las espinas hemales de las vértebras caudales 19-20. El primer pterigioforo proximal es ancho. Número total de vértebras: 34 .

Color en vivo: Descrita de una foto de un ejemplar vivo en un acuario mantenido 
alrededor de dos meses, al cabo del cual murió y se procedió a su fijación en formalina al $10 \%$. Cuerpo amarillo plateado sobre fondo azul oscuro. Una banda lateral amarillo plateado más intensa, que se extiende desde el margen posterior del opérculo hasta la base de la aleta caudal. Con mancha humeral oscura verticalmente alargada. Aletas pectoral y pélvicas rosadas, aletas dorsal, anal y caudal hialinas.

Color en alcohol: Cuerpo café-oscuro, mas pronunciado en la parte dorsal. Parte lateral del cuerpo con rayas café-oscuras estrechas y orientadas en forma oblicua que forman triángulos invertidos. Los ejemplares conservan guanina en la parte dorsal y lateral. Una mancha opercular oscura, sin macha humeral. Bordes expuestos de las escamas de la parte dorsal y lateral del cuerpo delineados por cromatóforos oscuros. Aleta caudal con una banda oscura en los radios medios. Aleta dorsal marcadamente pigmentada de color oscuro. Aleta anal levemente pigmentada en sus extremos. Cabeza oscura, con mayor intensidad en su parte dorsal, mas claro en la parte ventral.

Distribución: Conocida sólo en su localidad típica.

Aspectos ecológicos: Según Barriga y Ternaus (2005), las Lagunas El Voladero constituyen el principal ecosistema léntico de la reserva ecológica El Angel, están ubicadas sobre una llanura extensa donde el tipo de topografía y vegetación no permite una adecuada escorrentía, convirtiendo las zonas aledañas de las lagunas en verdaderos pantanos en cuyo paisaje domina Cyperaceas, Poaeceas y Juncaceae. La laguna central tiene una extensión de $72 \mathrm{~m}^{2}$. La profundidad en algunos sitios no sobrepasa los $3 \mathrm{~m}$ y en la parte media no mayor de $1.5 \mathrm{~m}$, en la orilla tiene una media de $0.80 \mathrm{~m}$ de sustrato limoso. Es una laguna oligotrófica. $\mathrm{pH} 5.5$, conductividad no supera los $15 \mu \mathrm{s} / \mathrm{cm}$, temperatura superficial oscila entre $10-17^{\circ} \mathrm{C}$. Los peces fueron capturados sobre la vegetación acuática de ribera.

Además, Barriga y Ternaus (2005) recomiendan que las lagunas se conserven en su estado natural ya que el sistema léntico no soportara impactos ambientales, tales como el uso del agua para riegos y otras actividades. Su hábitat es muy sensible a los cambios en el nivel del agua, ya que G. quitoensiss vive entre la vegetación acuática de las orillas, y al producirse éste efecto, podrían ser presa fácil de la trucha Oncorhynchus mykiss que fue introducida. Por lo tanto, solicitan eliminar la población de ésta especie introducida y realizar un monitoreo del nivel del agua en las lagunas.

Etimología: Epíteto especifico alusivo a la ciudad de Quito, en Ecuador

Comentarios: Con base en el análisis efectuado por Román-Valencia et al. (2003) las sinapomorfías observadas en este trabajo para el género Grundulus son las siguientes: ausencia de aleta adiposa, número reducido de escamas perforadas en la línea lateral; presencia de supraorbital y hueso pélvico delgado.

La forma y el tamaño en los tres taxones: $G$. bogotensis, G. cochae y G. quitoensis es igual, por lo tanto el análisis de componentes principales (ACP) llevado a cabo no es informativo.

\section{Clave taxonómica para las especies de Grundulus}

1. Aleta anal con cuatro a cinco radios simples; aletas pectorales con dos a tres radios simples; dentario con ocho a

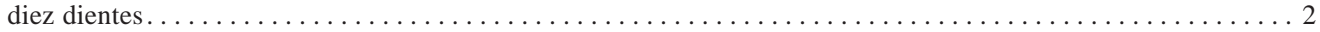

1.1. Aleta anal con tres radios simples; aletas pectorales con un radio simple; dentario con $12-14$ dientes

$\ldots \ldots \ldots \ldots \ldots \ldots \ldots \ldots \ldots \ldots \ldots \ldots \ldots \ldots \ldots \ldots \ldots \ldots \ldots \ldots \ldots \ldots \ldots$ quitoensis sp.nov.

2. Dentario con diez dientes; maxilar con siete a diez dientes; aletas pectorales con tres radios simples

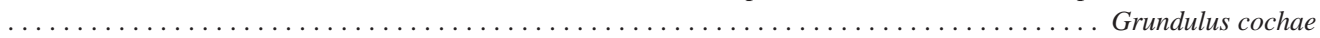

2.2. Dentario con ocho a nueve dientes; maxilar con cuatro a siete dientes; aletas pectorales con dos radios simples

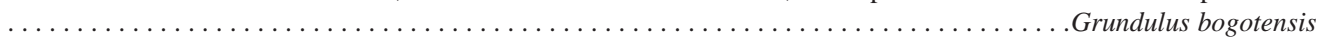




\section{AGRADECIMIENTOS}

La Universidad del Quindío (comité central de investigaciones) financió un viaje de estudio al Ecuador (C.R.-V. y R. I.R.). Idea Wild suministró reactivos utilizados en la diafanización de material. Plutarco Cala, Donald C. Taphorn y Flavio C.T. Lima por las correcciones y la lectura critica efectuada al manuscrito. La figura 1 y fue elaborado por D.C. Taphorn.

\section{RESUMEN}

Se describe Grundulus quitoensis nueva especie (Characiformes, Characidae) proveniente de las Lagunas El Voladero reserva biológica El Angel al norte de Ecuador. El nuevo taxon se distingue de las demás especies por el menor número de radios simples en la aleta anal (tres vs. cuatro a cinco) y en las aletas pectorales (uno vs. dos a tres), por el mayor número de dientes en cada dentario (1214 vs. 8-10), perfil ventral posterior a la cintura pectoral hasta el origen de la aleta anal plano (vs. curvo), el segundo infraorbital es tres veces más grande que el primero (vs. dos veces más grande), el tercer infraorbital está separado del preopérculo (vs. en contacto con la superficie del preopérculo), dentario presenta cuatro pequeñas fenestras en el margen infero-lateral (vs. uno a dos), el premaxilar presenta un proceso lateral largo (vs. corto), maxilar con dos muescas en la superficie infero-lateral (vs. maxilar con una muesca en la superficie inferolateral).

Palabras clave: Grundulus quitoensis sp. nov. Characidae, peces andinos; Sudamérica.

\section{REFERENCIAS}

Barriga, R.S. \& E. Ternaus. 2005. Primer hallazgo de una población paleoendémica del pez Grundulus cf. bogotensis (Humboldt,1821) en los altos Andes del Ecuador. Politécnica 26: 1-13.

Cala, P. \& C. Román-Valencia. 1994. Lista y distribución geográfica de las especies de la familia Characidae (Pisces, Characiformes) en Colombia. Biol. Educ. 4: 15-23.

Dahl, G. 1971. Los peces del norte de Colombia. Inderena, Bogotá. 391 p.
Eigenmann, C.H. 1915. The Cheirodontinae, a subfamily of minute characid fishes of South America. Mem. Carnegie Mus. 7: 1-99, pls 1-17.

Eigenmann, C.H. 1922. The fishes of the Northwestern South America including Colombia, Panama and Pacific slopes of Ecuador and Peru, together with appendix upon the fishes of the rio Meta in Colombia. Mem. Carnegie Mus. IX: 1-348.

Géry, J. 1977. Characoids of the world. T.F.H. Publ. Neptune City. 672 p.

Hubbs, C.L. \& K.F. Lagler. 1958. Fishes of the Great Lake Region. Revised edition, Cranbook Institute of Science, Bulletin 26, Bloomfield Hills, Michigan

Humboldt, F.H., A. Von \& A. Valenciennes. 1833. Reserches sur les poissons fluviatiles de l'Amerique equinoxiale En: Humboldt F.H. A. et A. Bonpland, d' observations de zoologie et d'anatomie Comparèe. Paris 2: 154-156, $159 \mathrm{pl} \mathrm{45,} \mathrm{fig.} 1$.

Malabarba, L.R. 1998. Monophyly of the Cheirodontinae: Characters and major clades (Teleostei: Characidae), p. 193-233. In L.R Malabarba, R.E. Reis, R.P. Vari, Z.M.S. de Lucena \& C.A.S. Lucena (eds.). Phylogeny and clasification of neotropical fishes. Edipucrs, Porto Alegre, Brasil.

Miles, C. 1971. Los peces del río Magdalena. U.T. Ibagué, Edic. (Reimpresión), 214 p.

Román-Valencia, C., Hans-Joachim Paepke \& F. Pantoja. 2003 (“2001”). Una especie nueva de Grundulus (Teleostei: Ostariophysi: Characidae) para Colombia y redescripción de Grundulus bogotensis (Humboldt y Valenciennes, 1833). Mem. Fund. La Salle Cienc. Nat. 155: 51-72.

Taylor, W.R. \& G.C. Van Dyke. 1985. Revised procedures for staining and clearing small fishes and other vertebrates for bone and cartilage study. Cybium 9: 107-119.

Vari, R.P. 1995. The neotropical fish family Ctenoluciidae (Teleostei: Ostariophysi: Characiformes) supra and intrafamilial phylogenetic relationships, with a revisionary study. Smith Contr. Zool. 564: 1-96.

Weitzman, S.H. 1962. The osteology of Brycon meeki, a generalized characid fish, with an osteological definition of the family. Stanford Icthyol. Bull. 8: 1-77.

Weitzman, S.H. \& L.R. Malabarba. 1999. Systematics of Spintherobolus (Teleostei: Characidae: Cheirodontinae) from eastern Brazil. Icthyol. Explor. Freshwaters 10: 1-43. 